\title{
ORIGINAL ARTICLE \\ Functional impact of multidisciplinary outpatient program on patients with chronic complete spinal cord injury
}

This article has been corrected since advance online publication and a corrigendum is also printed in this issue.

\author{
N Derakhshanrad ${ }^{1}$, F Vosoughi ${ }^{1}$, MS Yekaninejad ${ }^{2}$, P Moshayedi ${ }^{3}$ and H Saberi ${ }^{1,4}$
}

\begin{abstract}
Study design: Prospective study.
Objectives: The objective of this study was to determine whether an integrated and an intensive outpatient program would result in functional improvement of spinal cord injury (SCI) patients with American Spinal Injury Association Impairment Scale (AIS) A injuries as measured by the Spinal Cord Independence Measure (SCIM III).

Setting: Patient recruitment and evaluations were conducted at the Brain and Spinal Injury Repair Research Centre (BASIR), Tehran, Iran.

Methods: Observed SCIM III scores and SCI Ability Realization Measurement Index changes were used to measure the change in 134 patients with complete SCI (AIS A), after participation in an outpatient rehabilitation program consisting of a bimonthly multidisciplinary education program, combined with a twice-weekly occupational therapy, physical therapy and home nursing as a rehabilitation package for a 6 -month period.

Results: A significant increase in median total SCIM III scores following comparison of 'pre-treatment' scores and final 'post-treatment' scores $(9.5$ score, $P<0.001)$ was found. The increase in final SCIM III scores was highest in lower cervical ( 8.75 scores) and thoracic cases (13.5 scores). With the exception of high cervical patients, all subgroups had a significant SCIM III score improvement.

Conclusion: Multidisciplinary, outpatient rehabilitation programs are recommended as a safe and an effective post-injury rehabilitation for AIS A SCI patients. Such programs may complement inpatient rehabilitation and promote functional recovery. Multidisciplinary outpatient programs are effective in achieving long-term independence in $\mathrm{SCl}$ patients and reducing the cost of care for developing countries. This study suggests that high cervical injuries benefit more from inpatient programs.
\end{abstract}

Spinal Cord (2015) 53, 860-865; doi:10.1038/sc.2015.136; published online 4 August 2015

\section{INTRODUCTION}

Minor levels of auto-recovery are expected after occurrence of spinal cord injury (SCI), particularly in cases with incomplete lesions. Complete SCIs on the other hand have a considerably lower chance of auto-recovery, and acute rehabilitation measures are recommended, especially within the first 4 weeks after the injury. ${ }^{1,2}$ However, this acute rehabilitation may be delayed for various reasons. Major reasons for a delay in commencement of rehabilitation programs include a late presentation by the patient and a premature discharge with inadequate or no rehabilitation following initial hospitalization.

A major hurdle in the provision of rehabilitation programs in developing countries is the inadequacy of available facilities, especially for inpatient rehabilitation services. ${ }^{1}$ Accordingly, Ackerman et al. developed an outpatient program for patients with complete motor SCI. They used a multidisciplinary approach and demonstrated independence levels comparable with inpatient rehabilitation programs (in patients who had already undergone a brief inpatient program). 3,4 Outpatient day programs were considered to be extensions of inpatient rehabilitation, which helped reduce the length of hospitalization. These programs have uniformly used the Spinal Cord Independence Measure (SCIM) III scale as a reliable and a valid instrument to measure changes in functional outcomes. ${ }^{5-9}$

Determining the optimal SCIM III scores as a function of neurological level and severity of the injury is an important prerequisite for assessing efficacy of rehabilitation programs. ${ }^{10}$ To assess the quality of outcome regardless of the neurological level, the SCI Ability Realization Measurement Index (SCI-ARMI) has been developed as an independent assessment of functional gain after rehabilitation. ${ }^{10-12}$

Given the very low probability of auto-recovery in patients with American Spinal Injury Association (ASIA) impairment scale $\mathrm{A}^{13}$ choosing this group of patients minimizes the functional variation between cases due to auto-recovery, and helps attribute any changes to the rehabilitation interventions.

${ }^{1}$ Brain and Spinal Injury Repair Research Center (BASIR), Imam Khomeini Hospital, Tehran University of Medical Sciences, Tehran, Iran; ${ }^{2}$ Department of Epidemiology and Biostatistics, School of Public Health, Tehran University of Medical Sciences, Tehran, Iran; ${ }^{3}$ Department of Neurology, UCLA, Los Angeles, CA, USA and ${ }^{4}$ Department of Neurosurgery, Imam Khomeini Hospital, Tehran University of Medical Sciences, Tehran, Iran

Correspondence: Dr H Saberi, Brain and Spinal Injury Repair Research Center (BASIR), Imam Khomeini Hospital, Tehran University of Medical Sciences, Keshavarz Boulevard Postal Box 14185-61, Tehran 1449614535, Iran.

E-mail: hgsaberi@yahoo.com

Received 24 January 2015; revised 26 June 2015; accepted 1 July 2015; published online 4 August 2015 
The aim of our study is to measure the SCIM III scores as well as SCI-ARMI values in ASIA Impairment Scale A SCI patients before and following a 6-month rehabilitation intervention consisting of a bimonthly multidisciplinary outpatient education program, in addition to twice-weekly occupational therapy, physical therapy and home nursing.

\section{PARTICIPANTS AND METHODS}

\section{Participants}

This prospective study was conducted from April 2012 to December 2013 with 134 complete SCI patients selected from those referred to the Brain and Spinal Injury Repair Research Center (BASIR) affiliated to Tehran University of Medical Sciences. The patients were referred to BASIR as a tertiary center for SCI rehabilitation. At initial interview, the demographic and clinical data (including medical records, magnetic resonance imaging (MRI), computed tomography (CT) scan and X-ray images) were registered, and patients were classified according to the severity of injury. With permission from Institutional Review Board, patients aged between 18 and 60 years with ASIA impairment scale A, a traumatic cause for SCI, who had not received rehabilitation for at least a 6-month period and who provided informed consent were included in the study. Frankle grades were verified by two independent neurosurgeons at the first visit. Those with severe concomitant brain injury, clinically significant psychiatric or cognitive disorder, major disabling complications (for example, stage IV pressure sore, advanced heterotopic ossification in $>1$ joint) and those with a follow-up period of $<6$ months were excluded from the study. Major complications requiring inpatient treatment (one complicated pressure ulcer, one renal failure and urinary fistula, one hip osteomyelitis and one instrument failure) were admitted for inpatient services and treated outside the study. Application of the aforementioned criteria led to selection of 134 patients from a total of 450 individuals referred to the institute during the study time frame.

The neurological assessment results and prognosis were first discussed with the treatment team who then explained the diagnosis to the patients and their family. Possible emotional reactions and denials were managed by the neuropsychiatric team members including a clinical psychologist and a psychiatrist. The content of the multidisciplinary outpatient program was discussed with the patient, and written informed consent was obtained.

\section{Program description}

The program consisted of face-to-face education and group discussions for about $3 \mathrm{~h}$, which took place at the institute every 2 months, and twice-weekly therapy sessions, which took place in patients' homes or locality. The majority of the participants received the same number of sessions, with the exception of complicated cases who received an extra two sessions. The bimonthly sessions of education for self-care included: feeding, bathing, dressing and grooming. Respiratory issues and sphincter management were also covered (incentive spirometry, self-catheterization, bowel training and toileting). Patient mobility education included wheelchair skills, assistive devices and transfer techniques. Techniques for prevention of complications such as pressure sores and autonomic dysreflexia were also discussed with patients. Patients participated in private sessions (to discuss sexual problems) and group sessions lasting $\sim 3 \mathrm{~h}$ (5-6 in each group with separate sessions for tetraplegic and paraplegic patients) with a multidisciplinary team including neurosurgeon, urologist, coloproctologist, occupational therapist, physiatrist, nurse specialist and psychologist. Persian translations of the guidelines of American Spinal Cord Medicine Consortium were also provided for patients as booklets in an educational package (www.scipg.com). Physical and occupational therapists, as well as home nurses, conducted 2-h sessions twice weekly during this 6month period either in patients' homes or in the local health office.

\section{Assessment and data collection}

All the SCIM data have been reported according to the exact neurological level. In addition, the functional outcomes are reported according to the standardization of the grouping as recommended by Spinal Cord Journal. ${ }^{14}$

Periodic assessments including SCIM III score evaluation and detection of any new complications were performed bimonthly after multidisciplinary sessions. SCIM III scores were recorded before the program began and at the end of the final session. The development of new complications or mortality was recorded. During the program, clients were contacted by phone calls and questions were answered by physicians. To determine the achieved percentage of the expected SCIM III score, SCI-ARMI was calculated according to the following formula for each level:

$$
\mathrm{SCI}-\mathrm{ARMI}=\left(\mathrm{SCIM}_{\text {observed }} / \mathrm{SCIM}_{\text {highestvalue }}\right) \times 100
$$

In this formula according to Catz et. $a l^{10}, \mathrm{SCIM}_{\mathrm{observed}}$ refers to patient's actual SCIM III score, and SCIM highest value refers to the arithmetic mean of two highest observed SCIM III scores for each neurological level.

\section{Statistical methods}

Data are represented by medians and quartiles. Mann-Whitney $U$-test and Kruskal-Wallis statistical tests were used for comparing the total SCIM III scores and score gains according to neurological levels. The $\chi^{2}$-test of trend was used for testing the linear trend of final total score by lesion levels. A $P$-value $<0.05$ was considered to be statistically significant. Linear regression analysis was performed for estimating the effect of age, education years and neurological level on the final SCIM III scores. All tests were performed as two-sided. SPSS software (version 21, IBM Corporation, New York, NY, USA) was used for data analysis.

\section{RESULTS}

All 134 patients participated in the program regularly, with at most a 1-week deviation from the scheduled time frame. There were no missing data. Demographic data and lesion characteristics are presented in Table 1 . Nearly $78 \%$ of the participants were male $(\mathrm{M} / \mathrm{F}=3.5)$, and the median age upon admission was 27 years (range: 20-59). The median value for education within the sample was 10.0 years. The median time after injury was 16 months, and most injuries were between T7 and T12 (Table 1).

The median total SCIM III score on admission was 42.0 , whereas at the final evaluation this figure increased to 51.5. The 9.5-score improvement from admission to final evaluation was significant $(P<0.001)$. Median, first and third quartiles of final scores, and score

Table 1 Demographic and injury characteristics of patients

\begin{tabular}{ll}
\hline Variable & $\mathrm{N}(\%)$ \\
\hline
\end{tabular}

Age at admission in years

Median (Q1-Q3)

$27.0(21.0-34.8)$

Time after injury in days

Median (Q1-Q3)

$496(235.0-1066.5)$

Education in years

Median (Q1-Q3)

$10.0(8.8-12.0)$

Sex

Male

$104(77.6)$

Female

$30(22.4)$

Level of impairment

C1-4 8(6.0)

C5 $6(4.5)$

C6 $8(6.0)$

C7-8 $4(2.9)$

T1-6 $14(10.5)$

T7-12 $91(67.9)$

L1-S1 3 (2.2) 
Table 2 Median, first and third quartile (Q1-Q3) values of final SCIM III scores and SCIM III gains for the various SCI levels

\begin{tabular}{lcc}
\hline SCl level & Final SCIM III & \multicolumn{1}{c}{ SCIM III gain } \\
\cline { 2 - 3 } & Median (Q1-Q3) & Median (Q1-Q3) \\
& $12(8-15.8)$ & $1(0-5)$ \\
C1-4 & $18(12.3-27)$ & $4(2-5.3)$ \\
C5 & $22(18.5-30.8)$ & $4.5(2.3-9.5)$ \\
C6 & $29(19-53.3)$ & $13(5.5-14.5)$ \\
C7-8 & $21.5(17.5-33)$ & $5(2.8-10.5)$ \\
C5-8 & $48.5(41-62.3)$ & $13(4.5-21.5)$ \\
T1-6 & $57(45-64)$ & $14(7-22)$ \\
T7-12 & $73^{\mathrm{a}}$ & $12^{\mathrm{a}}$ \\
L1-S5 & $57(45-64)$ & $14(7-22)$ \\
T1-S5 & $51.5(35-61.8)$ & $9.5(4-16)$ \\
All & &
\end{tabular}

Abbreviations: SCl, spinal cord injury; SCIM, Spinal Cord Independence Measure. Bold values denote the total of non-bold variables above them.

a Because of the low number of cases in this level, first and third quartiles were not calculated.

Table 3 Median, first and third quartile (Q1-Q3) values of subscales' final SCIM III scores for various levels

\begin{tabular}{lcccc}
\hline SCl level & Self-care & RSM & Mobility $R T$ & Mobility IO \\
& range 0-20 & range 0-40 & range 0-10 & range 0-30 \\
\hline C1-4 & 0 (0-0) & $11(8-14.5)$ & $0(0-0)$ & $0(0-0)$ \\
C5 & $4(1-5.5)$ & $14(10.8-16)$ & $1(0-2.8)$ & $1(0-3.5)$ \\
C6 & $3(0-7.5)$ & $16(15.8-16.5)$ & $2(1.5-2.5)$ & $0(0-2.5)$ \\
C7-8 & $7(2.8-13.8)$ & $16.5(12-25.5)$ & $4(2-8.3)$ & $3(0.8-6)$ \\
C5-8 & $4(2-7)$ & $15.5(13.5-17.5)$ & $2(0.5-3.5)$ & $1(0-3)$ \\
T1-6 & $15(13-18)$ & $21.5(17.5-28.3)$ & $7(5-10)$ & $7(2.8-7.3)$ \\
T7-12 & $18(14-18)$ & $25(18.0-29.3)$ & $8(7-10)$ & $7(5-8)$ \\
L1-S1 & $18^{\mathrm{a}}$ & $28^{\mathrm{a}}$ & $10^{\mathrm{a}}$ & $8^{\mathrm{a}}$ \\
T1-S5 & $17(14-18)$ & $25(18-29)$ & $8(7-10)$ & $7(5-8)$ \\
All & $16(10-18)$ & $22(18-28)$ & $8(4.8-9)$ & $6(2.5-7.5)$ \\
\hline
\end{tabular}

Abbreviations: 10, indoor outdoor; RSM, respiration and sphincter management; RT, room toilet; $\mathrm{SCl}$, spinal cord injury; SCIM, Spinal Cord Independence Measure.

Bold values denote the total of non-bold variables above them.

aBecause of low number of cases in this level, first and third quartiles were not calculated.

gains for different neurological levels are presented in Table 2. There was a very small gain (one unit) in total SCIM III scores for upper cervical patients. This gain was substantial for C5,6 patients (between 4 and 5 units). The gain in total SCIM III scores was considerably higher in patients with $\mathrm{C} 7$ or lower levels of injury. There was no significant difference in the total SCIM III score gain between upper thoracic, lower thoracic and lumbar level patients $(P=0.22)$. The range for median gains in total SCIM III scores for these levels was between 12 and 14 units.

The final values and changes in SCIM III subscales are presented in Tables 3 and 4, respectively. There were no increases in the self-care (feeding, bathing, dressing and grooming) or mobility (room, toilet, indoors and outdoors) subscales for upper cervical level patients. There was no gain in median mobility scores for patients with SCI levels higher than C7. On the other hand, patients with the neurological level at or below C7 had a significant gain in sphincter management scores (5-8 units). Subjects with L1-S1 level of injury showed the greatest improvement in mobility (indoors and outdoors) and sphincter management (bowel and bladder) subscales (Table 4).
Table 4 Median, first and third quartile (Q1-Q3) values of subscales' SCIM III gain scores for various levels

\begin{tabular}{|c|c|c|c|c|}
\hline SCl level & $\begin{array}{l}\text { Self-care } \\
\text { range } 0-20\end{array}$ & $\begin{array}{c}R S M \\
\text { range } 0-40\end{array}$ & $\begin{array}{l}\text { Mobility } R T \\
\text { range } 0-10\end{array}$ & $\begin{array}{l}\text { Mobility } 10 \\
\text { range } 0-30\end{array}$ \\
\hline C1-4 & $0(0-0)$ & $1(0-5)$ & $0(0-0)$ & $0(0-0)$ \\
\hline C5 & $1(0-1.5)$ & $2(0-2.8)$ & $0(0-2)$ & $0(0-2)$ \\
\hline C6 & $1.5(0-2.3)$ & $3(0-8.5)$ & $0(0-0.5)$ & $0(0-0.5)$ \\
\hline C7-8 & $1(0-4.3)$ & $6(3.5-10.8)$ & $1.5(0.3-2)$ & $1(0-2.8)$ \\
\hline C5-8 & $1(0-2)$ & $2.5(0.25-6.5)$ & $0(0-2)$ & $0(0-2)$ \\
\hline T1-6 & $2.5(0-5)$ & $5(0-12.3)$ & $3(1.8-3.3)$ & $2(0-2)$ \\
\hline T7-12 & $1(0-4)$ & $8(3-13)$ & $1(0-3)$ & $1(0-4)$ \\
\hline L1-S1 & $0^{a}$ & $6^{a}$ & $1^{\mathrm{a}}$ & $3^{a}$ \\
\hline T1-S5 & $1(0-4)$ & $8(3-13)$ & $2(0-3)$ & $1(0-4)$ \\
\hline All & $1(0-4)$ & $6(1-11)$ & $1(0-3)$ & $1.5(0-3)$ \\
\hline
\end{tabular}

Abbreviations: IO, indoor outdoor; RSM, respiration and sphincter management; RT, room toilet; $\mathrm{SCl}$, spinal cord injury; SCIM, Spinal Cord Independence Measure.

Bold values denote the total of non-bold variables above them.

${ }^{a}$ Because of the low number of cases in this level, first and third quartiles were not calculated.

The median final scores for 19 specific SCIM III tasks for different SCI levels are presented in Table 5. The median final score in all specific tasks was zero for upper cervical patients, except for respiration (8 units). For all neurological levels, median score gains for stair management and ground-to-wheelchair transfers were zero. Maximum observed final scores for specific neurological levels are compared with final outcomes following classic inpatient rehabilitation ${ }^{4}$ and presented in Table 6.

Final SCIM III scores and gains based on neurological levels are presented in Figures 1a and b, respectively. As expected, the final SCIM III scores show an increasing trend for more caudal SCI levels $(P<0.001)$. Patients with C7 and lower levels of injury demonstrated more gains in total SCIM III scores, compared with upper neurological levels $(P=0.00003$; Figure $1 \mathrm{~b})$.

Multiple linear regression analysis was performed to assess the effect of education level, age and neurological status (tetraplegic vs paraplegic) on the final observed SCIM III scores. The neurological status, when adjusted for age and education level, had a significant positive effect on both the final and the highest observed SCIM III scores $(P<0.001)$. Age did not show a significant association with the mentioned variables $(P>0.7)$. Education level (in years) also showed a significant association with the final SCIM III scores $(P=0.005)$.

Final SCI-ARMI values for different neurological levels are shown in Figure 2. As expected, there was no significant linear correlation between the final SCI-ARMI values and the neurological level $(P=0.32)$. Table 7 presents the mean SCI-ARMI values for different neurological levels. Linear regression analysis revealed that age did not have any significant effect on the final SCI-ARMI values in participants $(P=0.311)$. The cumulative incidence of major complications at the first evaluation is tabulated in Table 8 . All the complications were managed medically on an outpatient basis. No mortality occurred during the study period.

\section{DISCUSSION}

Inpatient and outpatient approaches to SCI rehabilitation can complement each other in the management of SCI patient populations as discussed in the pertinent literature. ${ }^{3,4}$ Studies highlight the benefits of significant cost reductions and continued high-quality care by using a combination of inpatient and outpatient programs.

The outcomes of an outpatient day care program with 114 motor complete medically stable SCI patients with a mean disease duration of 


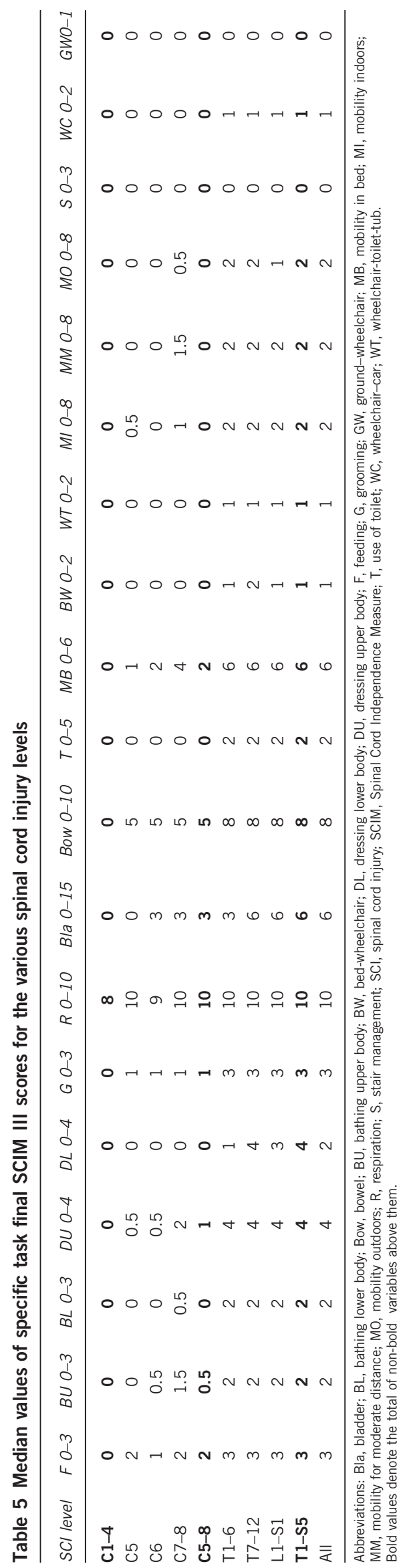

Table 6 Maximum final total SCIM III comparing outpatient and inpatient programs for various neurological levels

\begin{tabular}{|c|c|c|c|}
\hline \multirow[t]{2}{*}{ SCl level } & \multicolumn{2}{|c|}{ Outpatient (our study, $\mathrm{N}=134$ ) } & \multirow{2}{*}{$\frac{\text { Inpatient (Aidinoff et. al. }{ }^{4}, \mathrm{~N}=128 \text { ) }}{\text { SCIM III score }}$} \\
\hline & N & SCIM III score & \\
\hline C1-3 & 2 & 8 & 9 \\
\hline $\mathrm{C} 4$ & 6 & 16 & 36.5 \\
\hline C1-4 & 8 & 16 & 36.5 \\
\hline C5 & 6 & 33 & 32 \\
\hline C6 & 8 & 38 & 66 \\
\hline C7 & 3 & 59 & 38.5 \\
\hline C8 & 1 & 36 & 69 \\
\hline C5-8 & 18 & 59 & 69 \\
\hline T1-3 & 1 & 35 & 69.5 \\
\hline T4 & 2 & 45 & 80.5 \\
\hline T5 & 4 & 75 & 69.5 \\
\hline T6 & 7 & 63 & 69 \\
\hline T7 & 11 & 78 & 70 \\
\hline T8 & 2 & 70 & 70 \\
\hline T9 & 2 & 70 & 66 \\
\hline T10 & 23 & 72 & 74 \\
\hline T11 & 22 & 68 & 71 \\
\hline T12 & 31 & 82 & 76 \\
\hline L1 & 3 & 74 & 85.5 \\
\hline T1-S5 & 108 & 82 & 85.5 \\
\hline
\end{tabular}

Abbreviations: SCI, spinal cord injury; SCIM, Spinal Cord Independence Measure. Bold values denote the total of non-bold variables above them.

$\sim 3$ months have been previously reported in $2010 .^{3}$ Their average follow-up period was 17 days, and the provision of accommodation was required for the duration of the program. In our study, the multidisciplinary outpatient program consisted of three educational sessions provided at two-month intervals combined with physical and occupational therapy, as well as nursing interventions provided twice weekly in each patient's city of residence. Our final maximum SCIM III scores were compatible with scores previously reported in other studies $^{4}$ when adjusted for the neurological level (Table 6). As expected, upper cervical levels of injury do not benefit from outpatient programs, and inpatient protocols are more beneficial for them. Overall, a 9.5-unit improvement from admission to the final evaluation, approached 'substantial improvement' as defined by Scivoletto et al. ${ }^{15}$

Some studies report a negative impact of higher age on rehabilitation outcomes of SCI patients ${ }^{16}$. The median age of participants in this study was 27 (range 20-59) years. A negative effect of age on outcome was not shown $(P=0.7)$, although results indicate a decreasing trend for observed final SCIM III scores with increasing age.

The majority of participants took part in rehabilitation following a delay of at least 16 months. Despite the fact that this may have been expected to negatively affect the final outcomes, ${ }^{2,17}$ the increase in SCIM III scores was considerable. In this study, the importance of outpatient education even in late chronic SCI is highlighted (as the saying 'it's never too late to mend.'). Chhabra et. al ${ }^{14}$ compared 61 chronic SCI patients (mean chronicity $=9.3$ months) with 62 acute cases following participation in an inpatient program and reported a better outcome for acute cases. Early inpatient participation in acute rehabilitation programs, followed by outpatient multidisciplinary rehabilitation is recommended.

The sex ratio of our patients was similar to other studies. No relationship between sex and functional outcomes $(P=0.10)$ was 
Figure 1 Box plot for the relationship between neurological level groups and final total SCIM III score (a) and SCIM III gain score (b).

Figure 2 Final SCI-ARMI for different neurological levels.

found, and rehabilitation outcomes were person specific rather than gender specific ${ }^{11}$ as previously identified.

Regarding injury severity, we included only ASIA Impairment Scale A cases to reduce the confounding effect of auto-recovery on functional outcomes. For incomplete SCI cases, mathematical models have been proposed to predict the expected SCIM III scores, based on ASIA motor score for various ASIA Impairment Scales. ${ }^{10-12}$

Our patients consisted of $20 \%$ cervical cases compared with $\sim 50 \%$ in other outpatient-based studies. ${ }^{3}$ This discrepancy may be attributable to the fact that it is more difficult for cervical patients to participate in outpatient programs because of travel distance and/or problems with local accessibility in outpatient setups.

For both the cervical and the thoracic cases, the observed final SCIM III scores were predictably better at lower neurological levels compared with upper lesions. No mobility subscale improvement was identified in upper cervical ASIA Impairment Scale A patients. They improved in respiration and sphincter management subscales, consistent with previous reports of outpatient programs. Inpatient services are considered more beneficial for upper cervical patients (Table 6), ${ }^{3,4}$ For patients in every neurological level, stair management and ground-
Table 7 Mean final SCI-ARMI values for different neurological levels

\begin{tabular}{lcc}
\hline Lesion level & $\mathrm{N}$ & Mean final SCI-ARMI \\
\hline C1-3 & 2 & 100.00 \\
C4 & 6 & 81.83 \\
C1-4 & 8 & 86.37 \\
C5 & 6 & 53.33 \\
C6 & 8 & 51.88 \\
C7-8 & 4 & 71.05 \\
C5-8 & 18 & 56.62 \\
T1-4 & 3 & 93.49 \\
T5 & 4 & 108.00 \\
T6 & 7 & 69.14 \\
T7-9 & 15 & 62.60 \\
T10 & 23 & 62.65 \\
T11 & 22 & 75.95 \\
T12 & 31 & 61.26 \\
L1 & 3 & 91.67 \\
T1-S5 & 108 & 68.72 \\
Total & 134 & 74.96 \\
\hline
\end{tabular}

Abbreviation: SCI-ARMI, spinal cord injury-Ability Realization Measurement Index. Bold values denote the total of non-bold variables above them.

to-wheelchair transfer were very difficult to achieve, a finding consistent with other studies. ${ }^{3,4}$

In our patients, final SCIM III scores significantly increased with the descending neurological level. This finding may be perplexing for a rehabilitation team decision making when assessing the rehabilitation adequacy for various neurological levels. Correcting the absolute SCIM findings for SCI-ARMI would delete 'neurological level effect' on the final obtained SCIM III score (Figure 2), and this correction enables treatment team for better assessment of program adequacy.

\section{Limitations}

One of the apparent disadvantages of an outpatient approach is the necessity to travel to participate in a rehabilitation program. Methodologically, the lack of comparison with an inpatient group is a limitation of this study. This is mainly owing to the fact that only severely ill cases are admitted as inpatients in our hospital. Also, a small sample size for estimating the program effects in some 
Table 8 Morbidity in the studied cases

\begin{tabular}{lc}
\hline Medical complication & N (\%) \\
\hline ADR & $43(32.1)$ \\
& \\
Neuropathic pain (VAS, mm) & \\
$\quad \leqslant 50$ & $67(50.0)$ \\
$>50$ & $27(20.1)$
\end{tabular}

Active pressure sore (stage ${ }^{a}$ )

1

2

3

Heterotopic ossification

Hip

Knee

Spasticity (MAS)

1

2

3

Deep vein thrombosis (clinical data)

Minor depression (BDI)

Abbreviations: ADR, autonomic dysreflexia; BDI, Beck Depression Index; MAS, Modified Ashworth Scale; VAS, visual analogous scale.

aFour-stage system.

neurological levels was another limitation of this study. A further limitation is dependence on the education level and family support, that is, good family support was a prerequisite for a successful outcome in outpatient sessions. Nevertheless, outpatient sessions created a friendly atmosphere among the patients, most of them were willing to participate and happily pursue the program. It was also a motivation for promotion of self-care and social integration.

\section{CONCLUSION}

The current study demonstrates significant improvements in functional outcome for patients with complete injuries, with the exception of those with high cervical injuries, following a comprehensive outpatient rehabilitation program. It demonstrates the costeffectiveness of outpatient rehabilitation program as a complement to inpatient programs for patients without major complications. However, comprehensive inpatient rehabilitation may not always be available. Multidisciplinary outpatient programs are recommended as an effective but less costly method of providing rehabilitation for longterm achievement of independence among SCI patients, especially in developing countries.

\section{DATA ARCHIVING}

There were no data to deposit.

\section{CONFLICT OF INTEREST}

The authors declare no conflict of interest.

\section{ACKNOWLEDGEMENTS}

We thank Dr Seyed Hassan Emami Razavi, Dr Abolghasem Nikfallah, Dr Amir Hossein Tavakoli, Dr Mohammad Mohsen Maghari, Dr Parastoo Saberi and Asal Derakhshanrad for their kind support during the study. Also, our special thanks go to the unknown reviewers who have substantially improved the article by their sagacious suggestions.

1 Wyndaele M, Wyndaele JJ. Incidence, prevalence and epidemiology of spinal cord injury: what learns a worldwide literature survey? Spinal Cord 2006; 44: 523-529.

2 Scivoletto G, Morganti B, Molinari M. Early versus delayed inpatient spinal cord injury rehabilitation: an Italian study. Arch Phys Med Rehabil 2005; 86: 512-516.

3 Ackerman P, Morrison SA, McDowell S, Vazquez L. Using the Spinal Cord Independence Measure III to measure functional recovery in a post-acute spinal cord injury program. Spinal Cord 2010; 48: 380-387.

4 Aidinoff E, Front L, Itzkovich M, Bluvshtein V, Gelernter I, Hart J et al. Expected spinal cord independence measure, third version, scores for various neurological levels after complete spinal cord lesions. Spinal Cord 2011; 49: 893-896.

5 Anderson KD, Acuff ME, Arp BG, Backus D, Chun S, Fisher K et al. United States (US) multi-center study to assess the validity and reliability of the Spinal Cord Independence Measure (SCIM III). Spinal Cord 2011; 49: 880-885.

6 Bluvshtein V, Front L, Itzkovich M, Aidinoff E, Gelernter I, Hart J et al. SCIM III is reliable and valid in a separate analysis for traumatic spinal cord lesions. Spinal Cord 2011; 49: 292-296.

7 Catz A, Itzkovich M. Spinal Cord Independence Measure: comprehensive ability rating scale for the spinal cord lesion patient. J Rehabil Res Dev 2007; 44: 65-68.

8 Itzkovich M, Gelernter I, Biering-Sorensen F, Weeks C, Laramee MT, Craven BC et al. The Spinal Cord Independence Measure (SCIM) version III: reliability and validity in a multi-center international study. Disabil Rehabil 2007; 29: 1926-1933.

9 Catz A, Itzkovich M, Tesio L, Biering-Sorensen F, Weeks C, Laramee MT et al. A multicenter international study on the Spinal Cord Independence Measure, version III: Rasch psychometric validation. Spinal Cord 2007; 45: 275-291.

10 Catz A, Greenberg E, Itzkovich M, Bluvshtein V, Ronen J, Gelernter I et al. A new instrument for outcome assessment in rehabilitation medicine: Spinal Cord Injury Ability Realization Measurement Index. Arch Phys Med Rehabil 2004; 85: 399-404.

11 Scivoletto G, Glass C, Anderson KD, Galili T, Benjamin Y, Front L et al. An International Age- and Gender-Controlled Model for the Spinal Cord Injury Ability Realization Measurement Index (SCI-ARMI). Neurorehabil Neural Repair 2015; 29(I): 25-32.

12 Aidinoff E, Benjamini Y, Galili T, Polliack T, Front L, Bluvshtein V et al. Non-linear formulas for the spinal cord injury ability realization measurement index. Spinal Cord 2012; 50: 324-327.

13 Kirshblum SC, Burns SP, Biering-Sorensen F, Donovan W, Graves DE, Jha A et al. International standards for neurological classification of spinal cord injury (revised 2011). J Spinal Cord Med 2011; 34: 535-546.

14 Wyndaele JJ. Standardization of reporting data in the 'Spinal cord' journal. Spinal Cord 2012; 50: 483.

15 Scivoletto G, Tamburella F, Laurenza L, Molinari M. The spinal cord independence measure: how much change is clinically significant for spinal cord injury subjects. Disabil Rehabil 2013; 35: 1808-1813.

16 Hsieh CH, DeJong G, Groah S, Ballard PH, Horn SD, Tian W et al. Comparing rehabilitation services and outcomes between older and younger people with spinal cord injury. Arch Phys Med Rehabil 2013; 94: S175-S186.

17 Scivoletto G, Morganti B, Cosentino E, Molinari M. Utility of delayed spinal cord injury rehabilitation: an Italian study. Neurol Sci 2006; 27: 86-90. 